\title{
miR-124 Regulates the Phase of Drosophila Circadian Locomotor Behavior
}

\author{
Yong Zhang, ${ }^{1,2}$ Pallavi Lamba, ${ }^{1}$ Peiyi Guo, ${ }^{1}$ and Patrick Emery ${ }^{1}$ \\ ${ }^{1}$ Department of Neurobiology, University of Massachusetts Medical School, Worcester, Massachusetts 01605, and ${ }^{2}$ Department of Biology, University of \\ Nevada, Reno, Nevada 89557
}

Animals use circadian rhythms to anticipate daily environmental changes. Circadian clocks have a profound effect on behavior. In Drosophila, for example, brain pacemaker neurons dictate that flies are mostly active at dawn and dusk. miRNAs are small, regulatory RNAs $(\approx 22 \mathrm{nt})$ that play important roles in posttranscriptional regulation. Here, we identify $m i R-124$ as an important regulator of Drosophila circadian locomotor rhythms. Under constant darkness, flies lacking $m i R-124\left(m i R-124^{\mathrm{KO}}\right.$ ) have a dramatically advanced circadian behavior phase. However, whereas a phase defect is usually caused by a change in the period of the circadian pacemaker, this is not the case in $m i R-124^{\mathrm{KO}}$ flies. Moreover, the phase of the circadian pacemaker in the clock neurons that control rhythmic locomotion is not altered either. Therefore, $m i R-124$ modulates the output of circadian clock neurons rather than controlling their molecular pacemaker. Circadian phase is also advanced under temperature cycles, but a light/dark cycle partially corrects the defects in $m i R-124^{\mathrm{KO}}$ flies. Indeed, $m i R-124^{\mathrm{KO}}$ shows a normal evening phase under the latter conditions, but morning behavioral activity is suppressed. In summary, miR-124 controls diurnal activity and determines the phase of circadian locomotor behavior without affecting circadian pacemaker function. It thus provides a potent entry point to elucidate the mechanisms by which the phase of circadian behavior is determined.

Key words: circadian behavior; circadian rhythms; Drosophila; miRNAs

\section{Significance Statement}

In animals, molecular circadian clocks control the timing of behavioral activities to optimize them with the day/night cycle. This is critical for their fitness and survival. The mechanisms by which the phase of circadian behaviors is determined downstream of the molecular pacemakers are not yet well understood. Recent studies indicate that miRNAs are important regulators of circadian outputs. We found that $m i R-124$ shapes diurnal behavioral activity and has a striking impact on the phase of circadian locomotor behavior. Surprisingly, the period and phase of the neural circadian pacemakers driving locomotor rhythms are unaffected. Therefore, $m i R-124$ is a critical modulator of the circadian output pathways that control circadian behavioral rhythms.

\section{Introduction}

Most organisms use circadian clocks to anticipate daily environmental changes and thus maximize their chances of survival. Circadian

Received Sept. 1, 2015; revised Dec. 9, 2015; accepted Dec. $26,2015$.

Author contributions:Y.Z. and P.E. designed research;Y.Z., P.L., and P.G. performed research;Y.Z., P.L., P.G., and P.E. analyzed data; Y.Z. and P.E. wrote the paper.

This work was supported by the National Institutes of Health Grant GM100091 to P.E. and COBRE Grant P20 GM103650 to Y.Z. The content is solely the responsibility of the authors and does not necessarily represent the official views of the National Institutes of Health. We thank Eric Lai for the miR-124 ${ }^{\mathrm{K} 0}$ and genomic rescue flies and for sharing unpublished results; R. Stanewsky for the anti-PER antibody; the Developmental Studies Hybridoma Bank for anti-PDF antibody; and Chunyan Yuan and Diana Wentworth-Bilodeau for technical support.

The authors declare no competing financial interests.

Correspondence should be addressed to either of the following: Yong Zhang, Department of Biology, University of Nevada, Reno, 1664 N Virginia St, Reno, NV 89557, E-mail: yongzhang@unr.edu; or PatrickEmery, Department of Neurobiology, University of Massachusetts Medical School, 364 Plantation Street, Worcester, MA 01605, E-mail: patrick.emery@umassmed.edu. clocks govern most basic bodily functions, including sleep/wake cycles, hormone secretion, and metabolic rates. In animals, the molecular mechanism underlying circadian rhythms is a highly conserved transcriptional-translational feedback loop (Weaver and Emery, 2013). In flies, the heterodimeric transcription factor CLOCK/ CYCLE (CLK/CYC) binds to the promoter region of many genes through E-box sequences. Among these genes, period (per) and timeless (tim) encode for CLK/CYC repressors. PER and TIM form a heterodimer, accumulate in the cytoplasm, and enter into the nucleus to suppress their own gene transcription by inhibiting CLK/CYC. A series of kinases and phosphatases regulate the phosphorylation state, stability, and nuclear entry of PER/TIM. The oscillations of this molecular clock are maintained in constant 
conditions and synchronized by environmental inputs such as light and temperature. In flies, light changes the conformation of the bluelight photoreceptor CRYPTOCHROME (CRY). CRY binds to TIM and an E3-ubiquitin ligase containing JETLAG (JET) to trigger proteasomal TIM degradation (Zhang and Emery, 2012). TIM degradation exposes PER to phosphorylation and proteasomal degradation and thus resets the circadian pacemaker. Temperature increases also trigger TIM degradation to reset circadian clocks in flies, but this molecular response is mediated by calcium and the atypical protease SOL (Tataroglu et al., 2015). Circadian clock neurons can also receive thermal and photic inputs nonautonomously (HelfrichFörster et al., 2001; Chen et al., 2015).

miRNAs are small, noncoding RNAs that regulate many important biological processes through posttranscriptional repression of specific target genes (Bartel, 2004). Recent studies have shown that miRNAs play an important role in the control of circadian rhythms in both flies and mammals. Several miRNAs are reported to be under circadian control (Cheng et al., 2007; Yang et al., 2008; Vodala et al., 2012). In mice, two rhythmically expressed miRNAs are important for circadian clocks: miR-132 is induced by light and regulates circadian photoresponses and miR-219 modulates the circadian pacemaker (Cheng et al., 2007). Dicer proteins are critical for miRNA biogenesis (Bartel, 2004). In Dicer-deficient mice, shortened circadian rhythms have been observed mainly because of faster translation of PERIOD1 and PERIOD2, two critical pacemaker proteins (Chen et al., 2013). In flies, knocking down DICER-1 decreases the amplitude of locomotor rhythms (Kadener et al., 2009). We have also found that GW182, a protein crucial for miRNA function, controls locomotor rhythms through modulation of the pigmentdispersing factor (PDF) signaling pathway (Zhang and Emery, 2013). Recently, a cluster of miRNAs (miR959-miR964) was found to be under circadian regulation and to affect the timing of feeding, immune response, and circadian period (Vodala et al., 2012). In addition, miR-279 affects the amplitude of circadian behavioral rhythms (Luo and Sehgal, 2012), whereas bantam and let-7 control the expression of pacemaker genes (Kadener et al., 2009; Chen et al., 2014). Here, we reveal that miR-124 specifically modulates the phase of circadian locomotor behavior without affecting the circadian pacemaker of the brain neurons that control this complex behavior. In addition, we show that miR-124 affects morning activity and acute behavioral response to light.

\section{Materials and Methods}

Fly stocks. Flies were raised on standard cornmeal/agar medium at $25^{\circ} \mathrm{C}$ under light/dark (LD) cycles. The following strains were used: $w^{1118}$, $m i R-124^{\mathrm{KO}} / \mathrm{CyO}$ (Sun et al., 2012), miR-124 ${ }^{\mathrm{KO}}$, and genomic rescue $39 N 16 / C y O$ (Sun et al., 2012). The deficiency stocks BL7836 and BL7837 were obtained from the Bloomington Drosophila stock center.

Behavior experiments and analysis. Adult male flies ( $2-5 \mathrm{~d}$ old $)$ were used to test locomotor activity rhythms. Flies were entrained under LD for $3 \mathrm{~d}$ and released into constant darkness (DD) for at least $5 \mathrm{~d}$ at $25^{\circ} \mathrm{C}$. For temperature cycles (TCs), flies were entrained to $12 \mathrm{~h}: 12 \mathrm{~h} 29^{\circ} \mathrm{C}: 20^{\circ} \mathrm{C}$ thermocycle for $3 \mathrm{~d}$ in the dark and released at constant $25^{\circ} \mathrm{C}$ for at least $5 \mathrm{~d}$. Locomotor activity was recorded with Drosophila activity monitors (Trikinetics) in Percival I36-LL incubators. FaasX software was used to analyze behavioral data (Grima et al., 2002). For actograms, a signal-processing toolbox implemented in MATLAB was used (Levine et al., 2002). Three days of activity in LD were used to generate average activity bar graphs; $5 \mathrm{~d}$ of activity in DD were used to analyze phase.

Electroretinogram recordings. Electroretinograms (ERGs) were performed as described previously (Ni et al., 2008). Flies were immobilized with tapes. Glass recording and reference microelectrodes filled with Ringer's solution containing the following (in $\mathrm{mm}$ ): $130 \mathrm{NaCl}, 5 \mathrm{KCl}$, $2 \mathrm{CaCl}_{2}, 2 \mathrm{MgCl} 2,36$ sucrose, and 5 HEPES, pH 7.3, were inserted into small drops of electrode cream applied on the compound eye surface and the thorax, respectively. Light was provided by a $100 \mathrm{~W}$ halogen lamp and was delivered to the compound eye by fiber optics. Signals were amplified by a Warner IE210 intracellular electrometer, sampled, and analyzed using pCLAMP software. Five-second orange light pulses (1800 lux) were used to stimulate the eye after adapting the fly in the dark for $1 \mathrm{~min}$.

Whole-mount immunohistochemistry and quantification. Whole-mount immunohistochemistry was done as described previously (Lamba et al., 2014). For PDF stainings, flies were entrained to LD for $3 \mathrm{~d}$ and dissected at Zeitgeber time (ZT) 1 or 13. For PER stainings, flies were entrained to LD for $3 \mathrm{~d}$ and then released in DD. Brains were dissected on the second day of DD at six time points. Rabbit anti-PER (1:1500) and mouse anti-PDF (1:400) antibodies were used. All samples were imaged with a Zeiss LSM5 Pascal confocal microscope with a constant laser setting for each time point. ImageJ software was used for PER quantification. The average signal of three background areas were subtracted from signal intensity in each circadian neurons. At least five brains for each time point were used for quantification.

\section{Results}

\section{Loss of $m i R-124$ disrupts acute response to light in} the morning

Under an LD cycle, wild-type flies increase their activity before the lights-on (morning anticipation or morning peak) and before the lights-off transition (evening anticipation or evening peak) (Fig. 1A). Wild-type flies also show a sharp increase in activity at the lights-on and lights-off transition, which is called a startle response. These startle responses are direct reactions to environmental changes because they are independent of the circadian clock and happen even in otherwise arrhythmic flies $\left(\right.$ per $^{0}$ for example). However, the timing of morning and evening anticipation is under circadian clock control. The morning peak is driven by the PDF-positive small ventral lateral neurons (sLNvs), whereas the evening peak is driven by a subset of dorsal lateral neurons (LNds) and a PDF-negative sLNv (Grima et al., 2004; Stoleru et al., 2004). Dorsal neurons (DN1s) appear to work downstream of the sLNvs (Zhang et al., 2010a; Cavanaugh et al., 2014).

Because miR-124 is a highly conserved miRNA with specific expression in the CNS (Sun et al., 2012; Weng and Cohen, 2012), we decided to test whether it could influence circadian or diurnal behavior. $m i R-124^{\mathrm{KO}}$ flies showed a striking behavioral defect under an LD cycle: morning anticipation and the startle response to lights-on were severely blunted (Fig. 1A,B). However, an evening peak was clearly present under LD, albeit with reduced amplitude, suggesting that the molecular pacemaker is intact at least in the evening oscillators (Fig. 1B). To verify that this evening anticipatory behavior was indeed controlled by the circadian clock and was not merely a direct response to environmental input, we combined $m i R-124^{\mathrm{KO}}$ with the short period per ${ }^{\mathrm{S}}$ mutation (Konopka and Benzer, 1971). The phase of the evening peak was advanced in per ${ }^{\text {s }}$; $m i R-124^{\mathrm{KO}}$ double-mutant flies, as in per $^{\mathrm{S}}$ flies. This clearly demonstrates that the evening peak observed in $m i R-124^{\mathrm{KO}}$ flies is under circadian control (Fig. 1D).

To determine whether the phenotypes observed in LD are caused by loss of $m i R-124$, we first crossed $m i R-124^{\mathrm{KO}}$ flies to a deficiency line carrying a chromosome lacking the $m i R-124$ genomic region and tested heterozygous $m i R-124^{\mathrm{KO}} / \mathrm{Df}$ flies (Fig. 1A,B). These flies showed similar behavioral profiles as $m i R-124^{\mathrm{KO}}$ homozygous flies. The acute response to lights-on 

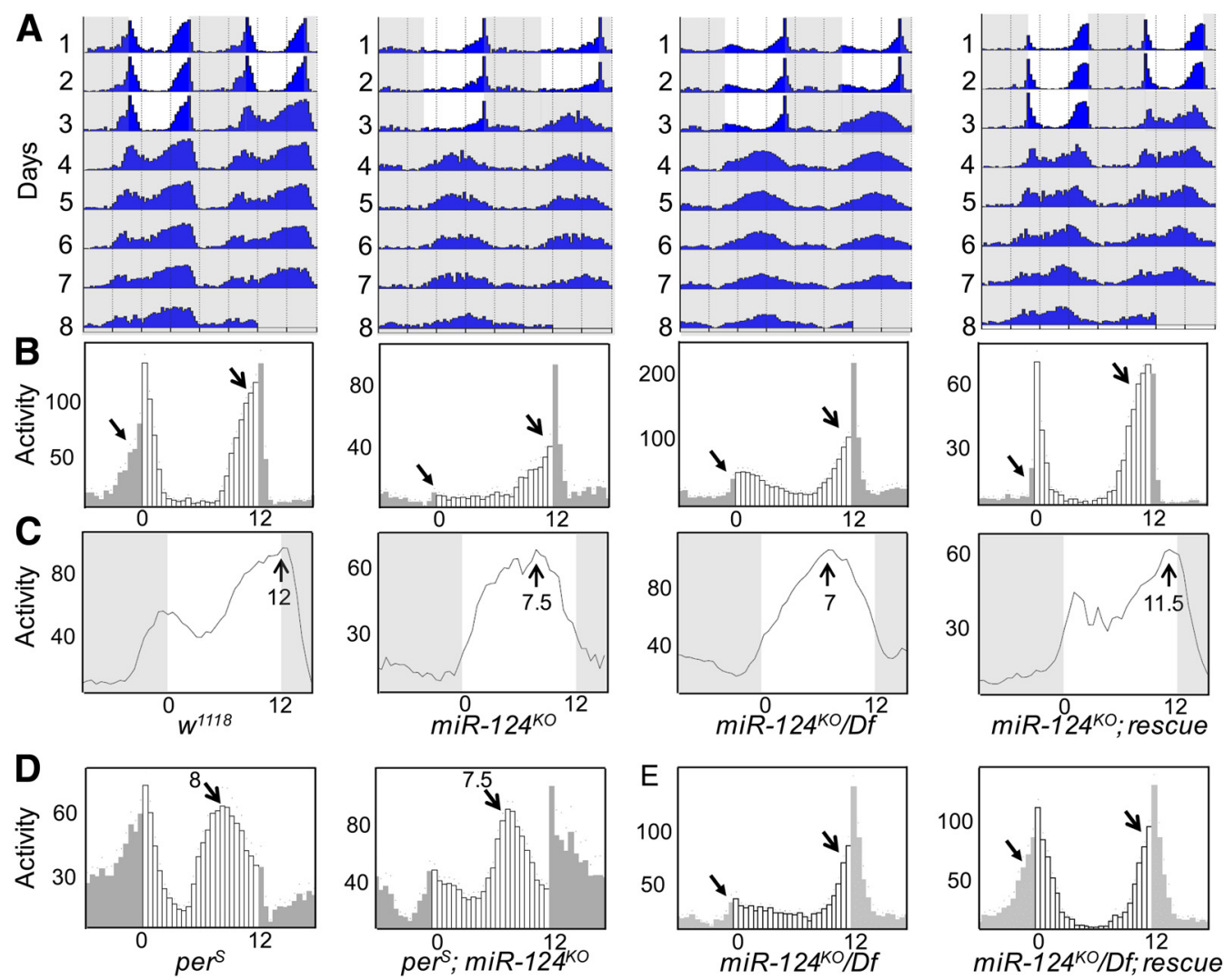

Figure 1. Loss of miR-124 advances circadian phase under constant darkness. A, Locomotor behavior under LD cycle and constant darkness. Representative double plotted actograms of $w^{1118}$, miR-124 ${ }^{\mathrm{K} 0}$, miR-124 ${ }^{\mathrm{K} 0} / \mathrm{Df}$, and miR-124 ${ }^{\mathrm{K} 0}$ rescue flies. White indicates the light phase, gray indicates the dark phase. $\boldsymbol{B}$, Morning anticipation (small arrows) and lights-on startle response are eliminated in miR-124 ${ }^{\mathrm{K} 0}$ flies under the LD cycle. Evening anticipation is indicated with large arrows. White bars represent activity during the day, gray bars at night. (C) Circadian behavior profile in DD. Circadian phase is dramatically advanced in $m i R-124^{\mathrm{K} 0}$ flies in constant darkness. Circadian time of peak activity is indicated on the graph. Gray shades indicate the subjective night. $\boldsymbol{D}$, Phase of the evening peak observed in miR-124 ${ }^{\mathrm{K} 0}$ flies is advanced by the $\mathrm{per}^{\mathrm{S}}$ mutation and is thus under circadian control. $\boldsymbol{E}$, Morning anticipation and lights-on startle response are restored in miR$124{ }^{\mathrm{KO}} / \mathrm{Df}$ flies rescued with a genomic miR-124 construct.

was absent. The amplitude of the morning peak of activity was reduced. We noted that the phase of the residual morning peak seemed delayed compared with wild-type. We then tested $m i R-124^{\mathrm{KO}}$ homozygous flies rescued with a genomic construct containing miR-124 (Sun et al., 2012; Fig. 1A,B). The morning startle response was restored, but the amplitude of morning anticipation showed little improvement. This is probably because of a general lower level of activity in $m i R$ $124^{\mathrm{KO}}$ homozygous flies carrying or not carrying the rescue transgene. $m i R-124^{\mathrm{KO}} / D f$ flies did not show this lower activity, which thus does not appear to be caused by loss of miR-124. Moreover, the morning peak of activity was restored in $m i R$ $124^{\mathrm{KO}} / \mathrm{Df}$ flies rescued with the genomic construct (Fig. $1 E$ ). In summary, $m i R-124$ is required for acute response to light in the morning and contributes to the timing and amplitude of morning anticipatory behavior.

\section{Loss of miR-124 advances circadian phase in constant darkness}

Under DD conditions, the morning and evening peaks of activity persist in wild-type flies with a phase determined by the previous LD (or temperature) cycle, but the amplitude of the morning peak tends to decrease over time. $m i R-124^{\mathrm{KO}}$ flies were rhythmic in DD with a completely normal period of $24.1 \mathrm{~h}$. To our surprise, however, the phase of circadian behavior was dramatically different in $m i R-124^{\mathrm{KO}}$ flies compared with wild-type flies. A single bout of activity was observed with a peak near subjective midday (Fig. 1C). This peak likely corresponds to a $4.5 \mathrm{~h}$ advanced evening peak of activity based on its sustained large amplitude and the fact that very little morning activity was detected under LD conditions in $m i R-124^{\mathrm{KO}}$. However, we cannot exclude a small contribution from morning neurons. A similar phenotype was observed in $m i R-124^{\mathrm{KO}} / D f$ flies. Both (subjective) morning and evening activity were restored with the genomic construct, with phases close to those observed in wild-type flies. Therefore, miR-124 plays an important role in the control of morning activity and determines the phase of evening activity under constant conditions.

\section{Loss of miR-124 advances circadian phase under and after temperature cycles}

Although the phase of evening activity was advanced in constant darkness, it was normal under the LD cycle (Fig. 1). Therefore, light input can correct this phase defect of miR$124^{\mathrm{KO}}$ flies. We therefore wondered whether temperature, another critical input to the clock, could do the same. We recorded the circadian behavior of $m i R-124^{\mathrm{KO}}$ flies under and after exposure to a $29^{\circ} \mathrm{C} / 20^{\circ} \mathrm{C}$ TC. To our surprise, unlike $\mathrm{LD}$, TC could not correct circadian phase. $m i R-124^{\mathrm{KO}}$ flies showed advanced evening phase under TC and after release to constant temperature (Fig. 2). Therefore, the phase defect is corrected specifically by light. 
Visual photoreception and the molecular pacemaker of circadian neurons are functional in $m i R-124^{\mathrm{KO}}$ flies

Because the startle response to the lights-on transition is blunted, we wondered whether visual photoreception is affected in $m i R-124^{\mathrm{KO}}$ flies. We therefore recorded the light response of visual photoreceptors to light with an ERG. The ERG of wild-type flies shows quick transients representing hyperpolarization and repolarization of laminar neurons postsynaptic to photoreceptors, as well as a sustained depolarization corresponding to the activation of the photoreceptor cells. No ERG defect was found in $m i R-124^{\mathrm{KO}}$ flies, indicating that the phototransduction cascade and synaptic transmission between photoreceptors and postsynaptic neurons in the eyes are normal (Fig. 3A). Therefore, the defect in light response in the morning is downstream of or unrelated to vision.

Next, we tested whether the advance in circadian behavioral phase could be caused by an abnormal phasing of the molecular pacemaker in the sLNvs, LNds, or DN1s, the three groups of circadian neurons known to control locomotor behavior. However, the amplitude and phase of PER oscillations were essentially normal (Fig. 3B,C). Therefore, miR-124 regulates circadian behavior phase by modulating circadian output mechanisms.

\section{Mild developmental abnormalities in the PDF neural network of $m i R-124^{\mathrm{KO}}$ flies}

We also wondered whether the development of pacemaker neurons controlling circadian behavior might be defective in $m i R$ $124^{\mathrm{KO}}$ flies. miR-124 has been reported to have a mild effect on neural development, especially bouton numbers at neuromuscular junctions (Sun et al., 2012; Wang et al., 2014). Therefore, we looked at the anatomy of PDF-positive neurons (Fig. 4). Indeed, PDF-positive sLNvs use PDF as a crucial neuropeptide for synchronizing other circadian neurons and to control morning activity (Renn et al., 1999). In all miR-124 ${ }^{\mathrm{KO}}$ brains, the expected set of LNvs with their projections was present: dorsal sLNv projection terminating in the dorsal protocerebrum and contralateral and optic lobe projections from the large LNvs (lLNvs) were observed (cf. Fig. $4 A-C, D-F$ ). However, in a small fraction $(22 \%)$ of $m i R-124^{\mathrm{KO}}$ brains, we observed one to two additional LNv projections. These either branched out of the sLNv dorsal projection bundle to terminate more ventrally than normal sLNv projections (Fig. $4 G$ ) or appeared to be $\mathrm{ILNv}$ projections dorsally branching out of the contralateral bundle toward the midline. miR-124 might thus have a minor impact on sLNv and lLNv projection pathfinding. We also noticed that the ILNv cell bodies tended to be positioned more dorsally than in wild-type flies (Fig. $4 A, D)$. Finally, we observed in a majority of $m i R-124^{\mathrm{KO}}$ brains $(68 \%)$ the persistence of projections from the tritocerebral PDF (PDF-Tri)-positive neurons (Fig. 4D, G), a noncircadian group of neurons that in wild-type flies is eliminated after eclosion through programmed apoptosis (Renn et al., 1999; Gatto and Broadie, 2011). Therefore, elimination of these cells is either delayed or defective in $m i R-124^{\mathrm{KO}}$ flies. As discussed below, the $m i R-124^{\mathrm{KO}}$ anatomical defects observed in the PDF network are unlikely to account for the behavioral phenotypes.

\section{Discussion}

We demonstrate here that miR-124 is crucial for regulating the phase of circadian behavior, as well as acute behavioral response to light. Unexpectedly, although circadian phase is advanced by up to $5 \mathrm{~h}$, the circadian pacemaker is unaffected. Its period is still $\sim 24 \mathrm{~h}$. Most mutants with a phase defect have altered circadian period. For example, the per $\mathrm{s}$ phase is considerably advanced under LD conditions, but this reflects the fast pace of the circadian pacemaker. Notable exceptions are mutants that affect per thermosensitive splicing, which helps flies adapt the phase of their evening peak to different ambient temperatures (Majercak et al., 1999). However, because PER oscillations are not affected at all in pacemaker neurons, it is highly unlikely that miR-124 regulates PER splicing. miR-124 thus works downstream of the circadian pacemaker to determine the circadian phase of locomotor behavior. It is therefore becoming increasingly clear that miRNAs play critical functions in the control of various circadian outputs. Indeed, miRNAs have also been implicated in the modulation of behavioral output amplitude (Luo and Sehgal, 2012), PDF signaling (Zhang and Emery, 2013), timing of feeding, and the immune response (Vodala et al., 2012). Interestingly, the latter rhythmic function was phase shifted in miR-959-miR964 cluster mutants, but whether this shift was caused by alterations 


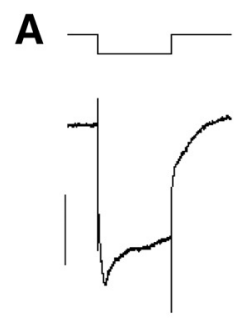

$W^{1118}$

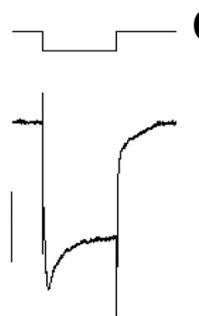

$\operatorname{miR}-124^{K O}$

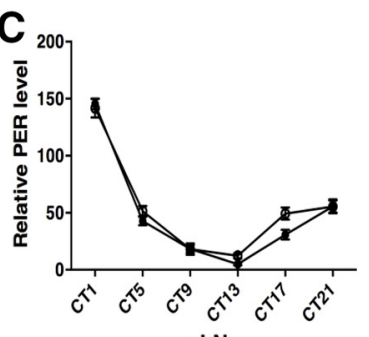

SLNv

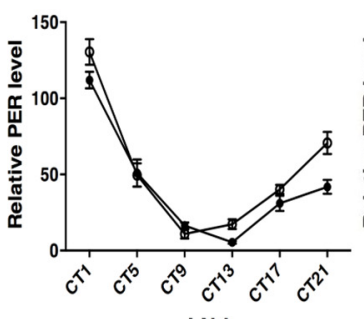

LNd

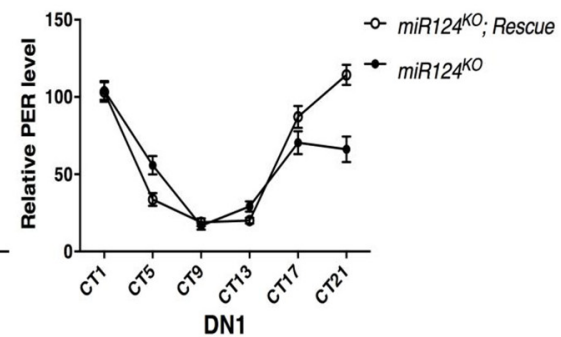

DN1

B

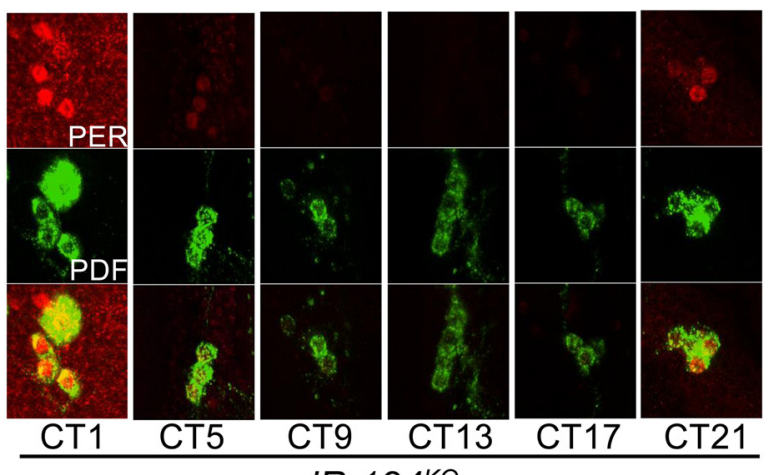

$\operatorname{miR}-124^{K O}$

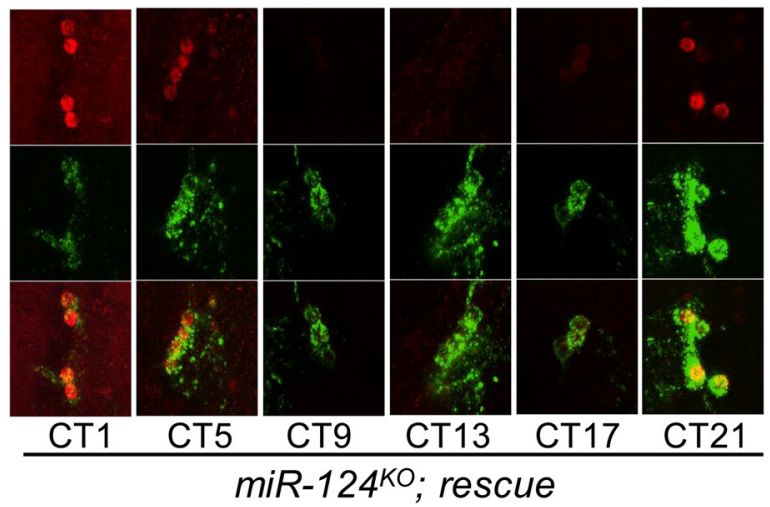

Figure 3. The molecular pacemaker is not affected in $m i R-124^{\mathrm{K} 0}$ flies. $A$, ERG recordings do not show any obvious light response defect in the visual photoreception cascade of miR-124 ${ }^{\mathrm{K} 0}$ flies. Scale bar, $5 \mathrm{mV}$. B, sLNvs of brains from miR-124 ${ }^{\mathrm{KO}}$ and genomic rescue flies dissected at different time points (circadian time, (T) during the second day of DD and stained with anti-PDF (green) and anti-PER (red) antibodies. C, Quantification of PER staining in sLNvs, LNds, and DN1s at different circadian time points. Error bars indicate SEM.
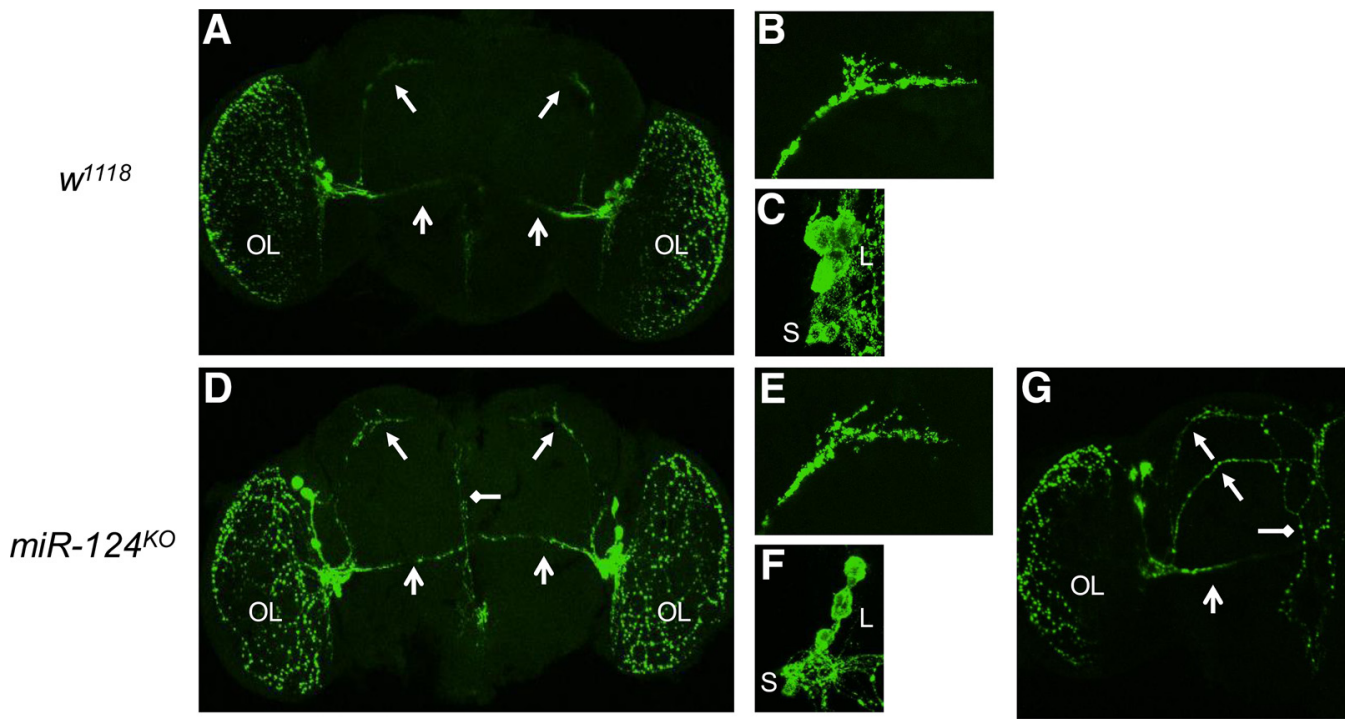

Figure 4. PDF neural network in wild-type and miR-124 ${ }^{\mathrm{K} 0}$ flies. PDF staining (green) in $w^{1118}(\boldsymbol{A}-\boldsymbol{C})$ and $m i R-124^{\mathrm{K} 0}(\boldsymbol{D}-\mathbf{G})$ brains. $\boldsymbol{A}, \boldsymbol{D}, \mathbf{G}, 0$ pen arrows indicate the sLNv dorsal projections, $0 \mathrm{~L}$ the ILNv optic lobes projections, and closed arrows the ILNv contralateral projections. These projections were all present and normal in miR-124 ${ }^{\mathrm{KO}}$ brains (D), but a small fraction of mutant brains showed additional LNv projections, such as more ventral sLNv projections (G). Persistence of PDF-Tri projections (diamond arrows) was observed in most mutant brains ( $\boldsymbol{D}, \boldsymbol{G}) . \boldsymbol{B}, \boldsymbol{E}$, Terminal ends of PDF-positive sLNv projections in the dorsal protocerebrum at ZT1. C, $F$, Cell bodies of PDF-positive sLNvs (S) and ILNvs (L).

of circadian pacemaker function or output pathways in relevant tissues is not yet known.

The task now is to define in which tissues miR-124 functions and which mRNAs it regulates. For the latter question, one can turn to target prediction. There are over 100 genes predicted by targetscan. In this list, a few targets are known to regulate circadian behavioral rhythms, including Mef2 and matrix metalloproteases (MMP1). Mef2 overexpression has been shown to lengthen circadian period, cause complex rhythms, and affect PER oscillations (Blanchard et al., 2010). The fact that we did not see such phenotypes makes it unlikely that miR-124 affected circadian behavior through Mef2. MMP1 has been shown to regulate circadian rhythms by affecting PDF levels at the dorsal terminals of sLNv neurons (Depetris-Chauvin et al., 2014). Acute overexpression of MMP1 during adulthood decreased PDF levels and the rhythmicity of flies in constant darkness. Again, these phenotypes do not fit those observed with $m i R-124^{\mathrm{KO}}$. Several positive components of BMP signaling pathway are targeted by miR-124 (Sun et al., 2012). However, constitutive activation of BMP pathway in circadian neurons caused a significant long period (Beckwith et 
al., 2013), whereas loss of $m i R-124$ had no effect on period. Identifying relevant $m i R-124$ targets is thus a priority and will require either genetic screening or gene expression profiling in relevant neurons.

This brings us to the important question of the site of $m i R$ 124 action. First, could the mild anatomical defects that we observed in PDF neurons account for the behavioral phenotypes? We cannot exclude this possibility, but believe it to be unlikely. The $1 \mathrm{LNv}$ defects were very subtle, with mostly a tendency to have more dorsally located cell bodies. In addition, the lLNvs do not control circadian behavior in DD nor are they required for morning activity and lights-on startle responses (Renn et al., 1999; Grima et al., 2004; Stoleru et al., 2004; Stoleru et al., 2005; Cusumano et al., 2009). Abnormalities in sLNv or $1 \mathrm{LNv}$ projections were too rare to account for the behavioral phenotypes that we observed. Finally, PDF-Tri cells, when preserved in adults through inhibition of apoptosis, have no impact on circadian behavior in LD or DD (Renn et al., 1999). Therefore, the persistence of these cells in $\mathrm{miR}$ $124^{\mathrm{KO}}$ flies again cannot explain the behavioral phenotypes. Finally, because light can correct the phase of $m i R-124^{\mathrm{KO}}$ flies in DD, a developmental defect appears unlikely to cause this phenotype. Interestingly, both the additional sLNv projections and the persistence of PDF-Tri cells are phenotypes observed in fmrl mutant flies (Gatto and Broadie, 2011). Moreover, FMR1 regulates $m i R-124$ levels (Xu et al., 2008). Combined with these previous studies, our current results thus suggest that the anatomical phenotypes observed in $\mathrm{fmr} 1$ mutants are at least in part caused by reduction in $m i R-124$ expression.

To map the anatomical requirement of $m i R-124$ for circadian behavior, we attempted to use a rescue approach with the GAL4/UAS system, but our efforts were unsuccessful, in part because overexpression caused unwanted phenotypes (including lethality). However, output from DN1s or neurons downstream of these circadian neurons are potential candidate sites for $m i R-124$ action. Indeed, the DN1s function downstream of the PDF-positive sLNvs and are critical for circadian rhythms in DD, as well as for morning activity and the lights-on startle response under LD (Zhang et al., 2010a; Cavanaugh et al., 2014). Interestingly, their output is modulated by light (Zhang et al., 2010b) and light was able to correct the phase defect of $m i R-124^{\mathrm{KO}}$ flies. TCs, however were, not able to do so, showing that a specific light input pathway reaches the neural circuit affected by $m i R-124$. Future work using more refined approaches to disrupt $m i R-124$ function or to restore it in a mutant background should identify this circuit and thus ultimately help to resolve the critical question of how circadian pacemaker and neural output are coupled.

\section{References}

Bartel DP (2004) MicroRNAs: genomics, biogenesis, mechanism, and function. Cell 116:281-297. CrossRef Medline

Beckwith EJ, Gorostiza EA, Berni J, Rezával C, Pérez-Santángelo A, Nadra AD, Ceriani MF (2013) Circadian period integrates network information through activation of the BMP signaling pathway. PLoS Biol 11: e1001733. CrossRef Medline

Blanchard FJ, Collins B, Cyran SA, Hancock DH, Taylor MV, Blau J (2010) The transcription factor Mef2 is required for normal circadian behavior in Drosophila. J Neurosci 30:5855-5865. CrossRef Medline

Cavanaugh DJ, Geratowski JD, Wooltorton JR, Spaethling JM, Hector CE, Zheng X, Johnson EC, Eberwine JH, Sehgal A (2014) Identification of a circadian output circuit for rest:activity rhythms in Drosophila. Cell 157: 689-701. CrossRef Medline

Chen C, Buhl E, Xu M, Croset V, Rees JS, Lilley KS, Benton R, Hodge JJ,
Stanewsky R (2015) Drosophila ionotropic receptor 25a mediates circadian clock resetting by temperature. Nature 527:516-520. CrossRef Medline

Chen R, D'Alessandro M, Lee C (2013) miRNAs are required for generating a time delay critical for the circadian oscillator. Curr Biol 23:1959-1968. CrossRef Medline

Chen W, Liu Z, Li T, Zhang R, Xue Y, Zhong Y, Bai W, Zhou D, Zhao Z (2014) Regulation of Drosophila circadian rhythms by miRNA let-7 is mediated by a regulatory cycle. Nat Commun 5:5549. CrossRef Medline

Cheng HY, Papp JW, Varlamova O, Dziema H, Russell B, Curfman JP, Nakazawa T, Shimizu K, Okamura H, Impey S, Obrietan K (2007) microRNA modulation of circadian-clock period and entrainment. Neuron 54:813-829. CrossRef Medline

Cusumano P, Klarsfeld A, Chélot E, Picot M, Richier B, Rouyer F (2009) PDF-modulated visual inputs and cryptochrome define diurnal behavior in Drosophila. Nat Neurosci 12:1431-1437. CrossRef Medline

Depetris-Chauvin A, Fernández-Gamba A, Gorostiza EA, Herrero A, Castaño EM, Ceriani MF (2014) Mmpl processing of the PDF neuropeptide regulates circadian structural plasticity of pacemaker neurons. PLoS Genet 10:e1004700. CrossRef Medline

Gatto CL, Broadie K (2011) Fragile X mental retardation protein is required for programmed cell death and clearance of developmentallytransient peptidergic neurons. Dev Biol 356:291-307. CrossRef Medline

Grima B, Lamouroux A, Chélot E, Papin C, Limbourg-Bouchon B, Rouyer F (2002) The F-box protein slimb controls the levels of clock proteins period and timeless. Nature 420:178-182. CrossRef Medline

Grima B, Chélot E, Xia R, Rouyer F (2004) Morning and evening peaks of activity rely on different clock neurons of the Drosophila brain. Nature 431:869-873. CrossRef Medline

Helfrich-Förster C, Winter C, Hofbauer A, Hall JC, Stanewsky R (2001) The circadian clock of fruit flies is blind after elimination of all known photoreceptors. Neuron 30:249-261. CrossRef Medline

Kadener S, Menet JS, Sugino K, Horwich MD, Weissbein U, Nawathean P, Vagin VV, Zamore PD, Nelson SB, Rosbash M (2009) A role for microRNAs in the Drosophila circadian clock. Genes Dev 23:2179-2191. CrossRef Medline

Konopka RJ, Benzer S (1971) Clock mutants of Drosophila melanogaster. Proc Natl Acad Sci U S A 68:2112-2116. CrossRef Medline

Lamba P, Bilodeau-Wentworth D, Emery P, Zhang Y (2014) Morning and evening oscillators cooperate to reset circadian behavior in response to light input. Cell Rep 7:601-608. CrossRef Medline

Levine JD, Funes P, Dowse HB, Hall JC (2002) Signal analysis of behavioral and molecular cycles. BMC Neurosci 3:1. CrossRef Medline

Luo W, Sehgal A (2012) Regulation of Circadian behavioral output via a MicroRNA-JAK/STAT circuit. Cell 148:765-779. CrossRef Medline

Majercak J, Sidote D, Hardin PE, Edery I (1999) How a circadian clock adapts to seasonal decreases in temperature and day length. Neuron 24: 219-230. CrossRef Medline

Ni L, Guo P, Reddig K, Mitra M, Li HS (2008) Mutation of a TADR protein leads to rhodopsin and Gq-dependent retinal degeneration in Drosophila. J Neurosci 28:13478-13487. CrossRef Medline

Renn SC, Park JH, Rosbash M, Hall JC, Taghert PH (1999) A pdf neuropeptide gene mutation and ablation of PDF neurons each cause severe abnormalities of behavioral circadian rhythms in Drosophila. Cell 99:791-802. CrossRef Medline

Stoleru D, Peng Y, Agosto J, Rosbash M (2004) Coupled oscillators control morning and evening locomotor behaviour of Drosophila. Nature 431: 862-868. CrossRef Medline

Stoleru D, Peng Y, Nawathean P, Rosbash M (2005) A resetting signal between Drosophila pacemakers synchronizes morning and evening activity. Nature 438:238-242. CrossRef Medline

Sun K, Westholm JO, Tsurudome K, Hagen JW, Lu Y, Kohwi M, Betel D, Gao FB, Haghighi AP, Doe CQ, Lai EC (2012) Neurophysiological defects and neuronal gene deregulation in Drosophila mir-124 mutants. PLoS Genet 8:e1002515. CrossRef Medline

Tataroglu O, Zhao X, Busza A, Ling J, O’Neill JS, Emery P (2015) Calcium and SOL protease mediate temperature resetting of circadian clocks. Cell 163:1214-1224. CrossRef Medline

Vodala S, Pescatore S, Rodriguez J, Buescher M, Chen YW, Weng R, Cohen SM, Rosbash M (2012) The oscillating miRNA 959-964 cluster impacts 
Drosophila feeding time and other circadian outputs. Cell Metab 16: 601-612. CrossRef Medline

Wang C, Feng T, Wan Q, Kong Y, Yuan L (2014) miR-124 controls Drosophila behavior and is required for neural development. Int J Dev Neurosci 38:105-112. CrossRef Medline

Weaver DR, Emery P (2013) Circadian timekeeping. In: Fundamental neuroscience (Squire LR, ed), pp 819-846. New York: Elsevier.

Weng R, Cohen SM (2012) Drosophila miR-124 regulates neuroblast proliferation through its target anachronism. Development 139:1427-1434. CrossRef Medline

Xu XL, Li Y, Wang F, Gao FB (2008) The steady-state level of the nervoussystem-specific microRNA-124a is regulated by dFMR1 in Drosophila. J Neurosci 28:11883-11889. CrossRef Medline

Yang M, Lee JE, Padgett RW, Edery I (2008) Circadian regulation of a lim- ited set of conserved microRNAs in Drosophila. BMC Genomics 9:83. CrossRef Medline

Zhang L, Chung BY, Lear BC, Kilman VL, Liu Y, Mahesh G, Meissner RA, Hardin PE, Allada R (2010a) DN1(p) circadian neurons coordinate acute light and PDF inputs to produce robust daily behavior in Drosophila. Curr Biol 20:591-599. CrossRef Medline

Zhang Y, Emery P (2013) GW182 controls Drosophila circadian behavior and PDF-receptor signaling. Neuron 78:152-165. CrossRef Medline

Zhang Y, Liu Y, Bilodeau-Wentworth D, Hardin PE, Emery P (2010b) Light and temperature control the contribution of specific DN1 neurons to Drosophila circadian behavior. Curr Biol 20:600-605. CrossRef Medline

Zhang Y, Emery P (2012) Molecular and neural control of insects circadian rhythms. In: Insect molecular biology and biochemistry (Gilbert LI, ed), pp 513-551. San Diego: Academic. 